\title{
Proton-Coupled Electron Transfer with Photoexcited Metal Complexes
}

\begin{tabular}{|r|l|}
\hline Journal: & Accounts of Chemical Research \\
\hline Manuscript ID: & ar-2012-00289x.R1 \\
\hline Manuscript Type: & Article \\
\hline Date Submitted by the Author: & 21-Nov-2012 \\
\hline Complete List of Authors: & Wenger, Oliver; University of Basel, Department of Chemistry \\
\hline \multicolumn{2}{|l}{} \\
\hline
\end{tabular}

\section{SCHOLARONE ${ }^{m}$ \\ Manuscripts}




\title{
Proton-Coupled Electron Transfer with Photoexcited
}

\section{Metal Complexes}

\author{
Oliver S. Wenger* \\ Universität Basel, Departement Chemie, Spitalstrasse 51, CH-4056 Basel, Switzerland
}

\section{CONSPECTUS}

Proton-coupled electron transfer (PCET) plays a crucial role in many enzymatic reactions and is relevant in the context of water oxidation, nitrogen fixation, and carbon dioxide reduction. Much contemporary research focuses on PCET between molecules in their electronic ground states, but PCET with photoexcited reactants receives increasing attention. This Account focuses on recent studies of excited-state PCET with $\mathrm{d}^{6}$ metal complexes with particular emphasis on work performed in the author's own group. Upon photoexcitation, some of these complexes release an electron and a proton to benzoquinone reaction partners; others act as combined electron-proton acceptors when phenols are present. This permits investigation of photoinduced PCET involving electron and proton transfer along a given direction, resembling hydrogen-atom transfer (HAT). In other studies the photoexcited metal complexes merely serve as electron donors or electron acceptors, whereas the proton donating and accepting sites are located on other parts of the molecular PCET ensemble. This multi-site design is used for exploration of so-called 
bidirectional PCET which plays an important role in many enzymes. Of pivotal interest in all of these studies is whether concerted proton-electron transfer (CPET) is kinetically competitive with sequential electron and proton transfer steps.
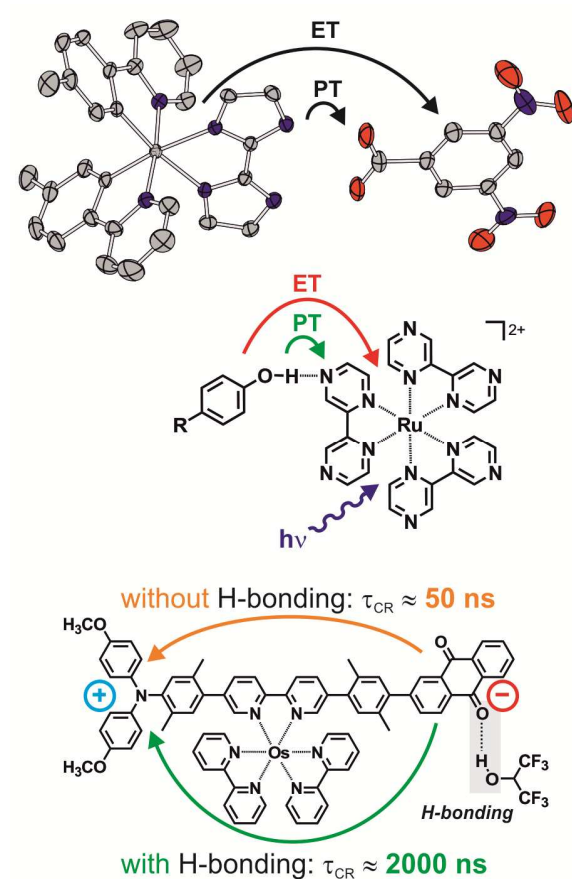

Excited-state PCET can be triggered by short laser pulses, and this makes investigation of rapid reactions possible. Luminescence spectroscopy is then a convenient tool to monitor PCET, but a combination with transient absorption spectroscopy is often desirable for unambiguous identification of reaction products. Nevertheless, in certain cases it may be tricky to distinguish between PCET photoproducts and reaction products formed simply by photoinduced electron transfer (ET), i. e., reactions not involving proton transfer (PT). Some of the studies presented herein deal directly with this important problem, for example one in which a cyclometalated iridium(III) complex is employed. Other studies with ruthenium(II) complexes and phenols focus on more or less systematic variations of the reaction free energies for the CPET, ET, and PT steps in order to explore what influence this might have on the overall PCET reaction. Yet other 
work with rhenium(I) complexes concentrated on the question how the electronic structure of the metal-to-ligand charge transfer (MLCT) excited states affects PCET. Covalent rhenium(I) phenol dyads were used to explore the influence of the electron donor - electron acceptor distance on bidirectional PCET. In covalent triarylamine-Ru(bpy $)_{3}{ }^{2+} / \mathrm{Os}(\mathrm{bpy})_{3}{ }^{2+}$-anthraquinone triads (bpy $=2,2$ '-bipyridine) hydrogen-bond donating solvents were found to cause significant lengthening of the lifetimes of photogenerated electron/hole pairs due to hydrogen-bonding to the quinone radical anion; the strengthening of H-bonds upon photoreduction is a variant of PCET which has received limited attention until now.

\section{INTRODUCTION}

Proton-coupled electron transfer (PCET) is of pivotal importance for activation of small inert molecules to more energy-rich substances. For example, the conversion of $\mathrm{CO}_{2}$ to formic acid is formally a two-electron / two-proton coupled reaction, ${ }^{1}$ the oxidation of water requires the accumulated loss of four electrons and four protons, ${ }^{2}$ while conversion of $\mathrm{N}_{2}$ to $\mathrm{NH}_{3}$ may even be regarded a coupled reaction of six electrons and six protons. ${ }^{3}$ When electrons and protons react in concerted fashion, charged high-energy intermediates can be circumvented and the overall chemical conversion can occur with more shallow activation barriers. ${ }^{4}$ In this sense, concerted proton-electron transfer (CPET) can be a significantly more favorable reaction pathway than a sequence of individual electron and proton transfer steps. Currently much fundamental research aims to elucidate under which circumstances CPET processes occur and how their rates and efficiencies can be optimized..$^{5-10}$ Numerous mechanistic studies focused on phenols because their oxidation is accompanied by a large increase in acidity, leading to the 
concerted release of an electron and a proton in many cases. ${ }^{5-7,11-18}$ Different experimental techniques have been used to trigger CPET with phenols, including the use of chemical oxidants, $^{11,16}$ electrochemical $^{8,13,14}$ or photochemical methods. $^{12,15,17-22}$ Ideally, the abovementioned small molecule activation processes would be driven by solar energy hence it might be useful to understand how light can be used to perform CPET chemistry. Much research performed so far has focused on CPET between molecules in their electronic ground-states, even when using photochemical flash/quench methods to trigger the reaction. ${ }^{15,17,18}$ Mechanistic studies of true excited-state PCET involving electronically excited molecules are comparatively scarce, but this sub-discipline of PCET has received increasing attention over the past couple of years. ${ }^{20,22-25}$ The theoretical framework is essentially the same irrespective of whether ground- or excited-state PCET reactions are investigated. However, excited-state PCET can be triggered by short laser pulses, and this permits investigation of rapid reactions and in many cases opens the possibility to use luminescence spectroscopy for monitoring the reaction.

Due to their long-lived ${ }^{3}$ MLCT (metal-to-ligand charge transfer) excited states $\mathrm{d}^{6}$ metal complexes with $\alpha$-diimine ligands have long been used for investigations of photoinduced charge and energy transfer. ${ }^{26}$ When equipped with protonatable or deprotonatable chemical functions, such complexes can become PCET reactants in their excited states. ${ }^{23,25}$ Sections 2 and 3 of this Account focus on recent mechanistic studies of PCET performed with such complexes in the author's group, put into the context of related work of other researchers. In section 4 multisite excited-state PCET between $\operatorname{Re}(\mathrm{I})$ complexes and phenol molecules is discussed, while section 5 concentrates on the influence of hydrogen-bonding on the lifetimes of charge-separated states formed after excitation of $\mathrm{d}^{6}$ metal complexes. Section 6 contains concluding remarks. 


\section{PHOTOEXCITED COMPLEXES AS COMINED ELECTRON/PROTON DONORS}

Early studies of excited-state PCET made use of amidinium-carboxylate salt bridges between $\mathrm{Ru}(\mathrm{bpy})_{3}{ }^{2+}$ (bpy $=2,2^{\prime}$-bipyridine) complexes and electron donors $/$ acceptors. $^{27,28}$ While electron transfer (ET) can easily be monitored in these systems, proton transfer (PT) across the salt bridge interface is more difficult to detect because the acid/base chemistry occurs only on the periphery of the chromophoric unit. Inspired by Fe(II) biimidazoline complexes which had been used successfully for investigations of ground-state PCET, ${ }^{4,29,30}$ photoactive $\mathrm{d}^{6}$ metal complexes of 2,2'-biimidazole were therefore investigated in the context of excited-state PCET. ${ }^{31-33} \operatorname{Ir}(\mathrm{III})$ complex 1 forms 1:1 adducts with dinitrobenzoate in $\mathrm{CH}_{2} \mathrm{Cl}_{2}$ (Figure 1); in the solid state there are two short $\mathrm{N}-\mathrm{O}$ distances indicative of hydrogen bonding between the imidazole $\mathrm{N}-\mathrm{H}$ functions and the carboxylate group. ${ }^{31}$

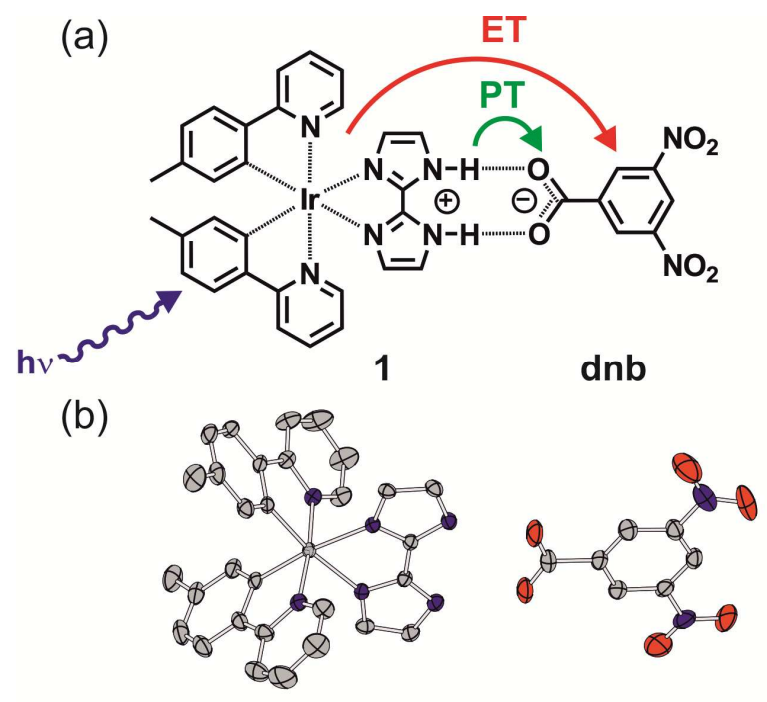

Figure 1. Salt-bridge adduct between complex 1 and 3,5-dinitrobenzoate (dnb). (a) Sketch of the photoinduced electron transfer (ET) and proton transfer (PT) processes. (b) Crystal structure of a cation-anion pair. ${ }^{31}$ 
From early studies by Haga it was known that biimidazole complexes of Ru(II) and Os(II) exhibit long-lived ${ }^{3}$ MLCT states from which electron and proton donation occurs more readily than from the ground state. ${ }^{34}$ Given the proximity of the N-H functions to the metal center it was hoped that deprotonation could be monitored by optical spectroscopy, and this was in fact one of the key motivations for this work. Luminescence spectroscopy was initially considered a particularly promising tool for this purpose. Indeed 3,5-dinitrobenzoate (dnb) quenches the emissive ${ }^{3}$ MLCT state of complex 1 oxidatively, and careful analysis of the luminescence spectra of 1 in presence of $\mathrm{dnb}$ reveals that ground and excited state potential energy surfaces are strongly displaced along a normal coordinate involving $\mathrm{N}-\mathrm{H}$ motion. ${ }^{31}$ To be more specific, in the emissive excited state substantial proton density is shifted from the biimidazole $\mathrm{N}$ atoms to the carboxylate $\mathrm{O}$ atoms hence this excited state is pre-organized to undergo PCET. However, it proved difficult to obtain much mechanistic insight from the biimidazole systems, and it seemed desirable to reduce the complexity of the excited-state PCET reagent by reverting to a $\mathrm{Ru}(\mathrm{II})$ complex (2) with a 2-pyridylimidazole ligand which has only a single deprotonatable N-H function. $^{35}$

Upon photoexcitation complex 2 is able to reduce 1,4-benzoquinone (BQ) (Figure 2), and this makes the $\mathrm{N}-\mathrm{H}$ group more acidic by roughly 3 orders of magnitude. ${ }^{36}$ In the course of reduction, the basicity of BQ increases by several orders of magnitude hence one might expect photoinduced electron transfer between 2 and BQ to be coupled to proton transfer. ${ }^{5}$ Indeed PCET photoproducts can be detected by transient absorption spectroscopy, but they form in low quantum yields because simple (= not proton-coupled) electron transfer is thermodynamically sufficiently favorable for rapid excited-state deactivation, and there is too little additional thermodynamic benefit from PCET. ${ }^{35}$ More electron-rich quinones would decelerate simple ET 
and would increase the chances of observing excited-state PCET. An investigation of groundstate PCET between a Ru(II) pyridylimidazole complex and the TEMPO radical was far more successful in providing mechanistic insight. ${ }^{37}$

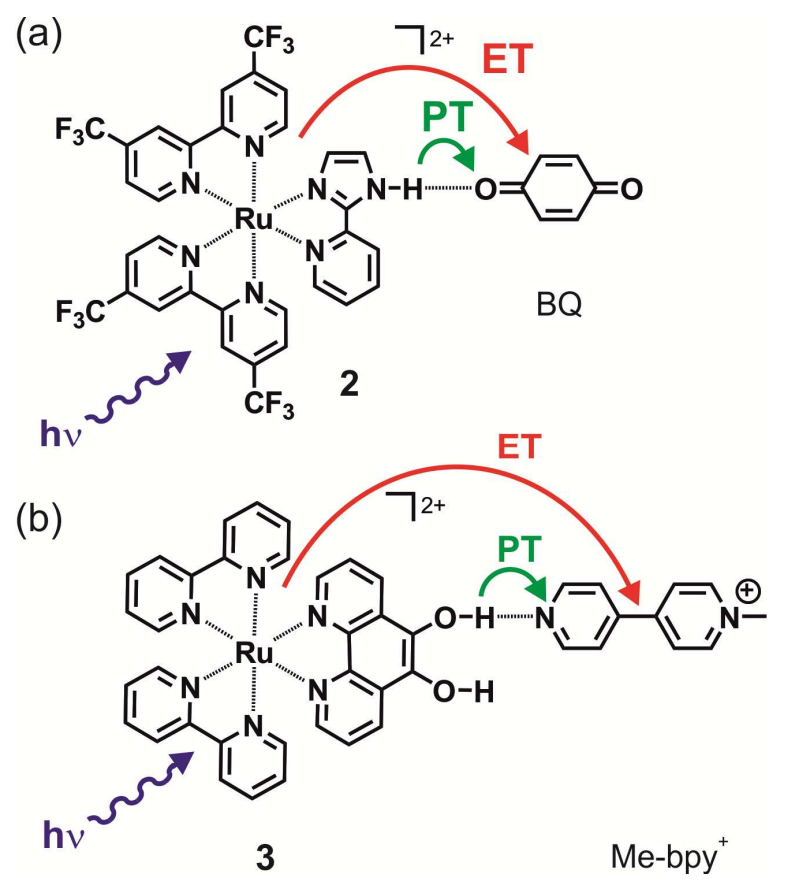

Figure 2. Combined photoacids/photoreductants with benzoquinone (BQ) and $N$ methylbipyridinium $\left(\mathrm{Me}-\mathrm{bpy}^{+}\right)$reaction partners. ${ }^{23,35}$

Complex 3 has deprotonatable $\mathrm{O}-\mathrm{H}$ groups and reacts with $\mathrm{N}$-methylbipyridinium (Me-bpy ${ }^{+}$) via CPET after photoexcitation (Figure 2). ${ }^{23} \mathrm{The} \mathrm{Me}-\mathrm{bpy}^{+}$reactant has the great advantage that PCET products can be detected unambiguously by transient absorption, and stepwise ET-PT or PT-ET reaction pathways can be ruled out on thermodynamic grounds. An important fundamental question which the studies presented in this section aimed to address is whether electron and proton movement in the course of an overall PCET reaction can be followed by monitoring independent spectroscopic handles. Provided sufficiently rapid excitation and detection techniques, a suitable system might provide very direct insight into the temporal 
evolution of a PCET process. Among the systems discussed in section 2, the combination of complex $\mathbf{3}$ and $N$-methylbipyridinium is the most promising in that regard.

\section{PHOTOEXCITED COMPLEXES AS COMBINED ELECTRON/PROTON}

\section{ACCEPTORS}

The redox and acid/base chemistry of $\mathrm{Ru}(\mathrm{II})$ complexes with 2,2'-bipyrazine (bpz) and 2,2'-bipyrimidine (bpm) ligands is well explored, ${ }^{38}$ and the comparatively large amount of available thermodynamic data provides a solid basis for investigation of their excited-state PCET reactivity. Using transient absorption and EPR spectroscopy Meyer and coworkers found that 1,4-hydroquinone reacts with photoexcited $\left[\mathrm{Ru}(\mathrm{bpy})_{2}(\mathrm{bpz})\right]^{2+}$ (4) via CPET, forming the reduced and protonated metal complex in addition to semiquinone (Figure $3 \mathrm{a}$ ). ${ }^{39,40}$

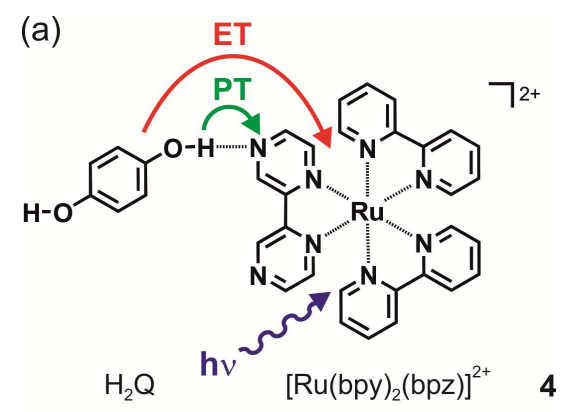

(b)

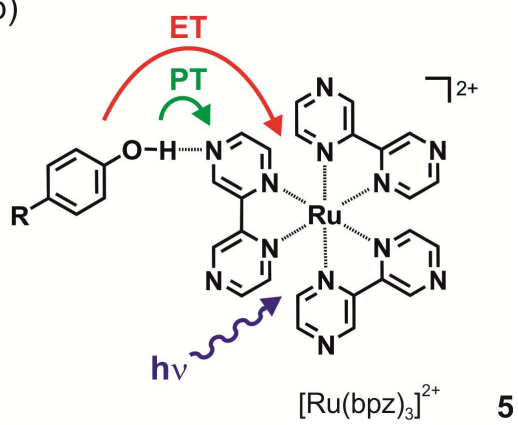


Figure 3. $\mathrm{Ru}(\mathrm{II})$ 2,2'-bipyrazine (bpz) complexes as combined electron/proton acceptors in photoreactions with 1,4-dihydroquinone $\left(\mathrm{H}_{2} \mathrm{Q}\right)$ and simple phenols $\left(\mathrm{R}=\mathrm{OCH}_{3}, \mathrm{CH}_{3}, \mathrm{H}, \mathrm{Cl}, \mathrm{Br}\right.$, $\mathrm{CN}){ }^{39,41}$

In related experiments we explored the photochemistry of $\mathrm{Ru}(\mathrm{bpz})_{3}{ }^{2+}(\mathbf{5})$ with 5 phenols bearing different para-substituents (Figure 3b). ${ }^{41}$ The rate constant for ${ }^{3}$ MLCT deactivation $\left(\mathrm{k}_{\mathrm{Q}}\right)$ by the phenols correlates with the $\mathrm{O}-\mathrm{H}$ bond dissociation free energy (BDFE) (Figure 4a), suggesting that all 5 phenols react with photoexcited $\mathrm{Ru}(\mathrm{bpz}){ }_{3}{ }^{2+}$ via CPET. There is a similarly good correlation between $\mathrm{k}_{\mathrm{Q}}$ and the phenol oxidation potentials (Figure $4 \mathrm{~b}$ ) which would be in line with ET as a rate-determining reaction step, but the observation of significant H/D KIEs for 4 out of 5 phenols seems incompatible with an ET-PT reaction sequence. There is no correlation of phenol $\mathrm{pK}_{\mathrm{a}}$ values with $\mathrm{k}_{\mathrm{Q}}$ (Figure $4 \mathrm{c}$ ), making a PT-ET sequence unlikely as well. $^{42}$ Interestingly, the H/D KIE increases with increasing electron-withdrawing character of the phenol para-substituent (R), ranging from 1.0 for $\mathrm{R}=\mathrm{OCH}_{3}$ to 10.2 for $\mathrm{R}=\mathrm{CN}$. The correlation between KIE and the nature of $\mathrm{R}$ is not understood. The fact that 4-methoxyphenol fits the correlation between $\mathrm{k}_{\mathrm{Q}}$ and the $\mathrm{O}-\mathrm{H}$ BDFE might suggest that a CPET mechanism is operative despite the absence of any significant H/D KIE in this particular instance. ${ }^{42}$

(a)

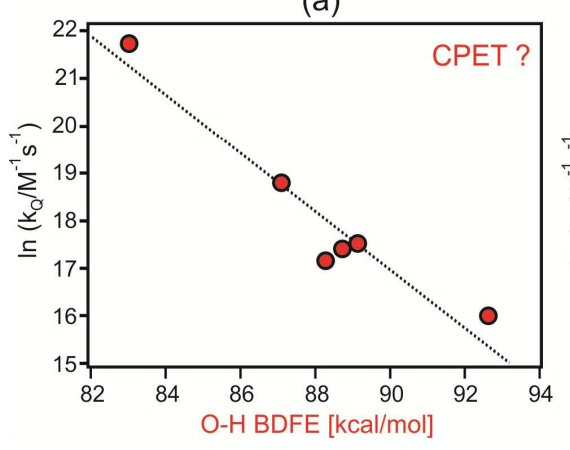

(b)

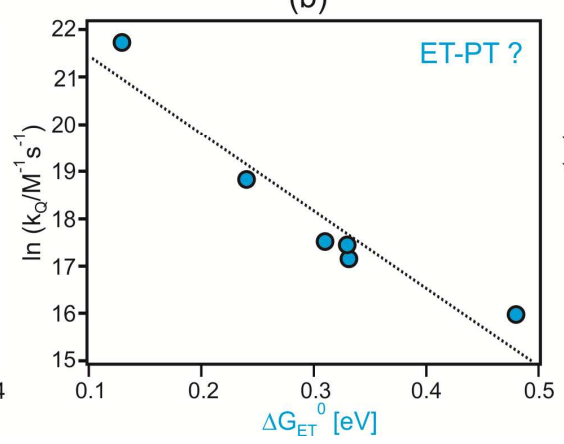

(c)

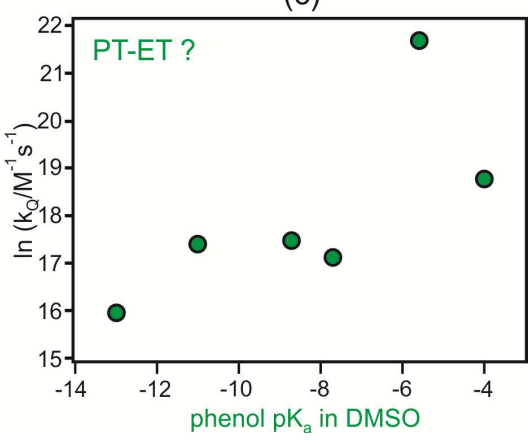


Figure 4. (a) Correlation of the rate constant for excited-state quenching $\left(\mathrm{k}_{\mathrm{Q}}\right)$ of $\mathrm{Ru}(\mathrm{bpz})_{3}{ }^{2+}(\mathbf{5})$ with the $\mathrm{O}-\mathrm{H}$ bond dissociation free energy (BDFE in DMSO) of 5 different para-substituted phenols; (b) correlation of $\mathrm{k}_{\mathrm{Q}}$ and the free energy associated with photoinduced ET from the phenols to complex $5 ;^{41}$ (c) correlation of $\mathrm{k}_{\mathrm{Q}}$ and the $\mathrm{pK}_{\mathrm{a}}$ values of the 5 phenols in DMSO. The thermodynamic parameters were taken from ref. ${ }^{5}$.

Conceptually, there is much analogy between this investigation of excited-state PCET ${ }^{41}$ and previous studies of ground-state PCET with phenols. ${ }^{11-19}$ Here, the use of a ruthenium(II) complex permits application of luminescence spectroscopy as a convenient detection tool. The following study addresses a fundamental question which is more specific to excited-state PCET chemistry.

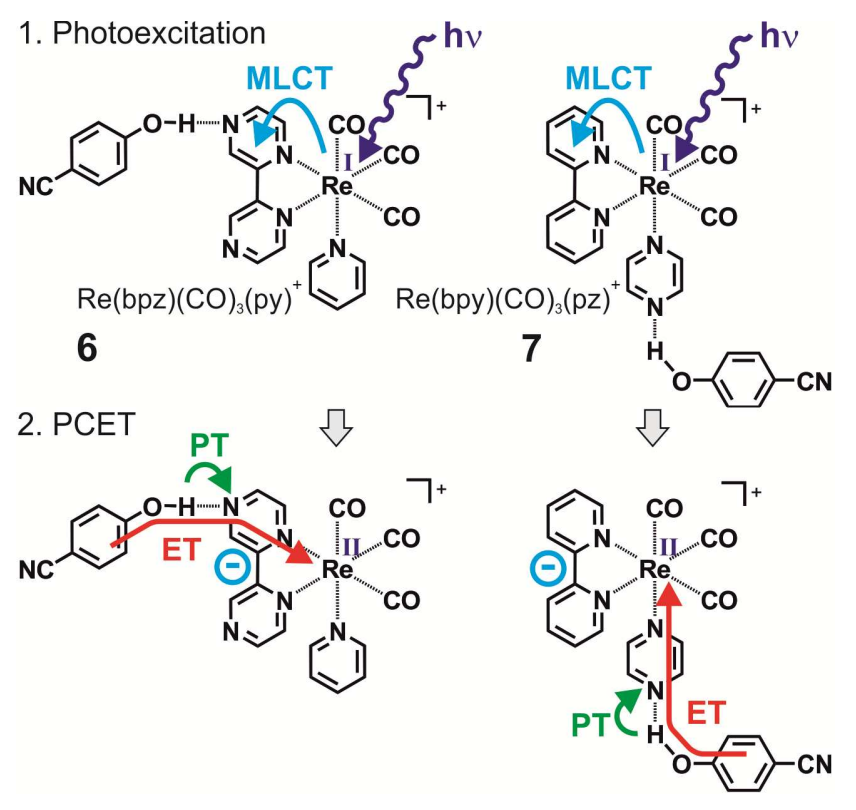

Figure 5. MLCT excitation and photo-PCET reactions in two rhenium(I) complexes with different protonatable sites. Reprinted with permission from ref. ${ }^{43}$. Copyright 2012 American Chemical Society. 
Using rhenium(I) tricarbonyl complexes the influence of the directionality of MLCT excitation on the photoinduced PCET chemistry with 4-cyanophenol was explored. ${ }^{43}$ Complexes 6 and 7 have protonatable bpz and 1,4-pyrazine (pz) ligands, respectively, and it appears reasonable to assume that in $\mathrm{CH}_{3} \mathrm{CN}$ solution 4-cyanophenol forms hydrogen-bonded encounter adducts with the peripheral $\mathrm{N}$ atoms of the bpz and pz ligands (upper half of Figure 5). In complex 6 the MLCT-excited electron is located on the bpz ligand, making its peripheral $\mathrm{N}$ atoms more basic than in the ground state. This is favorable for proton donation from the phenol but at the same time the MLCT-excited electron is in the middle of the ET pathway from phenol to the metal center (Figure 5, bottom left). In complex 7 the MLCT-excited electron is located on the bpy ligand which is beneficial for ET because there is now a direct ET pathway without Coulomb barrier (Figure 5, bottom right). However, in complex 7 the basicity of the pz ligand is decreased upon MLCT-excitation, making PT a less favorable process. Transient absorption spectroscopy provides direct evidence for the 4-cyanophenoxyl radical and it is clear that excited-state PCET takes place with both complexes. ${ }^{43} \mathrm{H} / \mathrm{D}$ KIEs and thermodynamic considerations are in line with a concerted reaction in both cases. The rate constant for excited-state deactivation via CPET is nearly identical for the two complexes, and thus the directionality of the MLCT excitation has little influence on the overall reaction kinetics in this case. This might be due to the fact that each of the two reaction couples has one favorable and one unfavorable contribution to the overall PCET reaction when thinking in thermodynamic cycles ("square schemes"). ${ }^{4}$ With complex 6 PT is favorable while ET is comparatively unfavorable, with complex 7 the opposite is the case. 


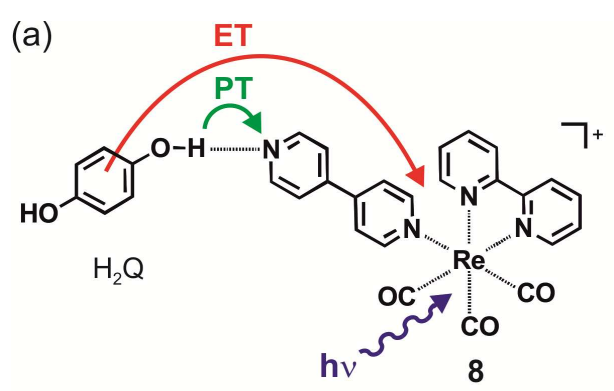

(b)

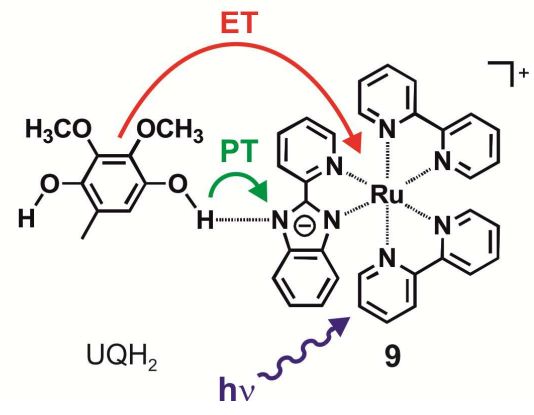

Figure 6. Excited-state PCET systems with protonatable 4,4'-bipyridine (a) or pyridylbenzimidazole (b) ligands. ${ }^{44,45}$

Complex 8 differs from 7 by the presence of a 4,4'-bpy instead of a pz ligand, and this makes protonation of the metal complex more easily detectable by transient absorption. This favorable property formed the basis for investigation of PCET reaction pathways between photoexcited 8 and 1,4-hydroquinone $\left(\mathrm{H}_{2} \mathrm{Q}\right)$ (Figure 6). ${ }^{44}$ Depending on conditions two different reaction mechanisms were found to be active. The first mechanism operates at low $\mathrm{H}_{2} \mathrm{Q}$ concentrations (in 1:1 $\mathrm{CH}_{3} \mathrm{CN} / \mathrm{H}_{2} \mathrm{O}$ mixtures) and is comprised of an ET-PT reaction sequence resulting from the photoexcitation of isolated (i. e., non-hydrogen-bonded) complexes; the rate-determining ET step leads to an H/D KIE of 1.1. At high $\mathrm{H}_{2} \mathrm{Q}$ concentrations $(>0.1 \mathrm{M})$ in the same solvent a substantial percentage of rhenium complexes are hydrogen-bonded to $\mathrm{H}_{2} \mathrm{Q}$ at the moment when they are excited by the laser pulse, and these encounter adducts react via CPET with an H/D KIE of 1.7. The overall PCET reaction 
mechanism thus crucially depends on the presence of hydrogen-bonded encounter adducts, and this is attributable to the short-range nature of proton transfer.

An investigation of PCET between an ubiquinol analogue $\left(\mathrm{UQH}_{2}\right)$ and a photoexcited Ru(II) complex bearing a deprotonated 2-pyridylbenzimidazole ligand (9) (Figure 6) provided insight into the oxidation of ubiquinol by the mitochondrial cytochrome bc $_{1}$ complex. $^{45}$ Complex 9 is chemically closely related to complex $\mathbf{2}$, the main conceptual difference is that 9 is used in its deprotonated form to act as a combined proton/electron acceptor whereas complex 2 is a combined photoacid/photoreductant. Excited-state deactivation of 9 by $\mathrm{UQH}_{2}$ occurs via CPET, manifesting an H/D KIE of 1.87. Stepwise ET-PT and PT-ET pathways were ruled out on thermodynamic grounds, and the semiquinone CPET product (UQH.) was detected by EPR. Indeed, EPR can a powerful technique for distinguishing simple redox products from PCET products. ${ }^{40}$

\section{BIDIRECTIONAL PCET WITH PHOTOEXCITED COMPLEXES}

PCET plays an important role in many enzymes, ${ }^{46-48}$ and the $\mathrm{P}_{680}{ }^{+} / \mathrm{Tyr}_{\mathrm{Z}} /$ His-190 reaction triple of photosystem II is an example which has received particular attention. ${ }^{49}$ This reaction triple exhibits bidirectional ground-state PCET, i. e., from the phenolic function of $\mathrm{Tyr}_{\mathrm{Z}}$ the electron is transferred to $\mathrm{P}_{680}{ }^{+}$while the proton is released to His-190. Numerous artificial model systems mimicking the PCET chemistry of the abovementioned triple have been explored, ${ }^{15,17-}$ ${ }^{20,50}$ and $\mathrm{d}^{6}$ metal complexes were frequently used to photo-trigger the overall reaction. However, in many cases a flash/quench technique was employed in order to generate highly oxidizing 
species which would then initiate a ground-state PCET reaction. ${ }^{15,17,18,21,51}$ A notable early exception is the study of phenol oxidation by photoexcited $\mathrm{C}_{60}$ in presence of pyridine, which revealed that electron transfer from phenol to the excited fullerene occurs in concert with release of the phenolic proton to the pyridine base. ${ }^{19}$

More recently, efforts to observe bidirectional excited-state PCET focused on covalent rhenium(I)-phenol dyads. The rhenium(I) tricarbonyl complex in molecule $\mathbf{1 0}$ is a strong photooxidant capable of triggering intramolecular phenol-to-rhenium ET in the excited state, and this is coupled to release of the phenolic proton to aqueous solution (Figure 7). ${ }^{22,52}$ The drivingforce for photoinduced intramolecular ET is sufficiently strong in this system to make an ET-PT reaction sequence the preferred pathway, and a concerted reaction is comparatively inefficient for this particular dyad. In this context, numerous studies (involving mostly species in their electronic ground states) concentrated on the question how PCET rates and mechanisms depend on $\mathrm{pH}$ and buffer concentration in aqueous solution. ${ }^{13-15,22,53}$ A recent study with dyad 11 explored excited-state PCET in $\mathrm{CH}_{2} \mathrm{Cl}_{2}$ in presence of pyridine and imidazole, and it was found that the overall reaction rate depends strongly on the strength of the base in this aprotic solvent, ${ }^{54}$ in agreement with prior studies of ground-state PCET. ${ }^{55}$ 

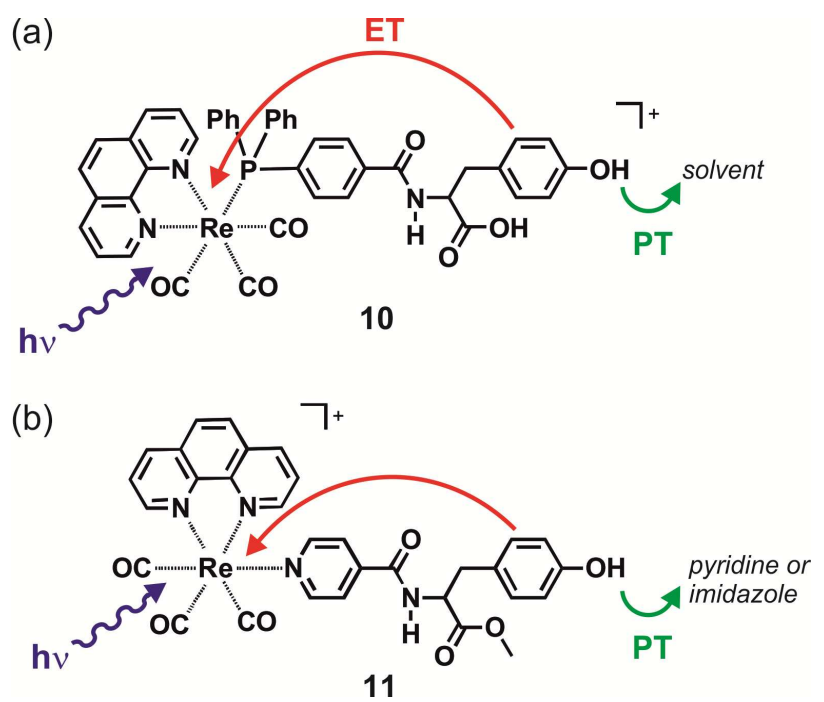

Figure 7. Artificial models mimicking the function of the $\mathrm{P}_{680}{ }^{+} / \mathrm{Tyr}_{\mathrm{Z}} /$ His-190 reaction triple of photosystem II. $^{22,54}$

An important fundamental question in the field of PCET is how the overall reaction depends on the electron and proton transfer distance. ${ }^{56-58}$ Using dyads $\mathbf{1 2}$ and $\mathbf{1 3}$ the dependence of excited-state PCET on the distance between the electron donor (phenol) and the electron acceptor (rhenium) was explored, a 1:1 mixture of $\mathrm{H}_{2} \mathrm{O} / \mathrm{CH}_{3} \mathrm{CN}$ served as proton accepting medium (Figure 8). ${ }^{59}$ Dyad 12 is an electronically strongly coupled donor-acceptor compound, whereas $\mathbf{1 3}$ is an electronically more weakly coupled donor-bridge-acceptor molecule. Transient absorption spectroscopy reveals that $\mathbf{1 2}$ acts principally as a photoacid. Photoinduced proton release to the solvent is accompanied by charge-reorganization on the phenol-substituted pyridine ligand to afford a tautomeric quinonoid form (Figure 8c) with negative charge transferred toward the metal center. This pyridone-like form seems to be more stable than a pyridine-phenolate tautomer. When a $p$-xylene unit is incorporated between the phenol and the pyridine the photochemistry changes, and dyad 13 exhibits excited-state PCET occurring through an ET-PT sequence. The ET step is rate-determining hence no significant H/D KIE is associated 
with the formation of photoproducts, but thermal back reaction to re-form the initial starting material occurs with $\mathrm{KIE}=3.7 \pm 0.5$, compatible with CPET or a PT-ET sequence.

(a)

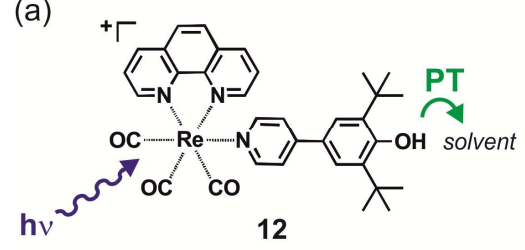

(d)

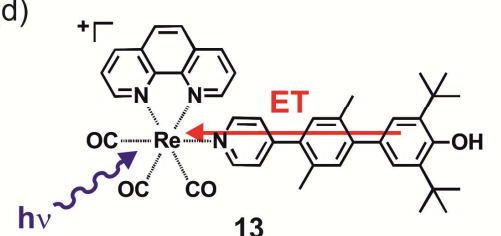

(b)

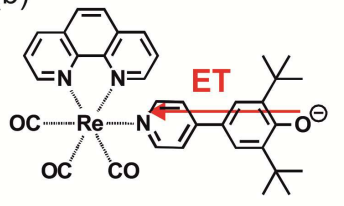

(e)

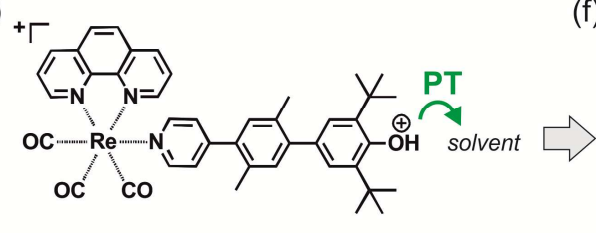

(c)

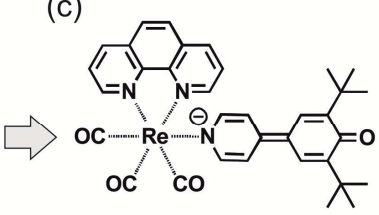

(f)

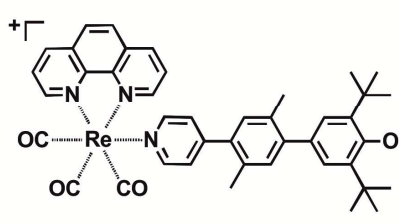

Figure 8. Influence of electron donor - electron acceptor distance on the photochemistry of rhenium(I)-phenol dyads in $1: 1 \mathrm{CH}_{3} \mathrm{CN} / \mathrm{H}_{2} \mathrm{O} .^{59}$

These two dyads illustrate nicely the close relationship between the fields of excited-state PCET and excited-state proton transfer. ${ }^{60}$ Dyad 12 represents an electronically strongly coupled donor-acceptor system that might be better described by the term "photoacid". Dyad 13, by contrast, is a weakly coupled donor-bridge-acceptor molecule for which it is more appropriate to speak of individual reaction partners (rhenium acceptor vs. phenol donor) participating in separate electron and proton transfer reactions. 


\section{HYDROGEN-BOND STRENGTHENING IN THE COURSE OF PHOTOINDUCED ET}

The presence of hydrogen bonds to (pendant or free) bases enables a CPET mechanism for phenol oxidation in many cases. ${ }^{6,8,11,12,14-16,21,51,57,61}$ Similarly, reduction of quinones is facilitated when hydrogen-bond donors are present. ${ }^{5,35,62,63}$ Given the importance of quinones as primary and secondary electron acceptors in bacterial photosynthesis there has been much work on artificial porphyrin-quinone dyads, ${ }^{26,64}$ but the aspect of hydrogen-bonding or proton-coupling has received surprisingly little attention in such studies. ${ }^{65}$ A famous exception is triad 14 in which photoexcitation of the porphyrin leads to a charge-separated state containing oxidized carotenoid and reduced quinone, with the latter being protonated by the pendant carboxylic acid function (Figure 9). ${ }^{66,67}$ Another early study of excited-state PCET with quinone acceptors made use of triplet-excited $\mathrm{C}_{60}$ which was oxidized by chloranil, coupled to proton donation from trifluoroacetic acid to reduced chloranil. ${ }^{68}$

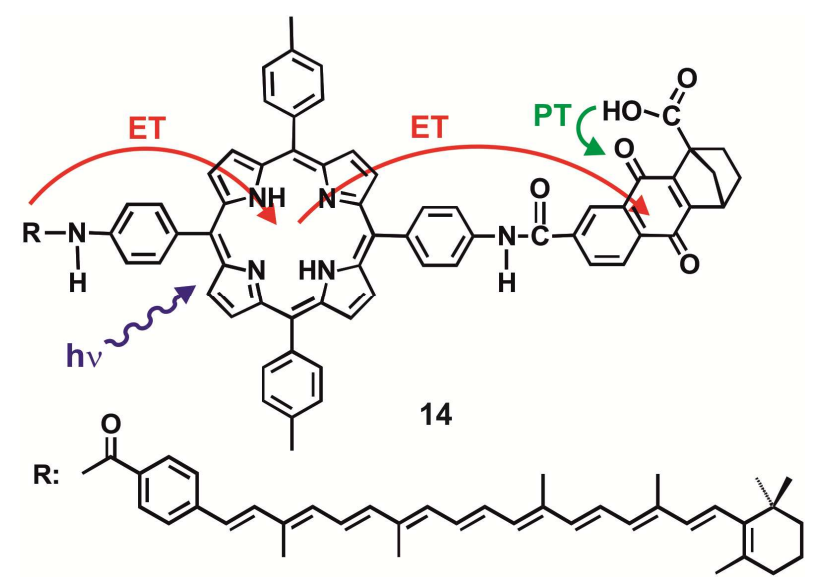

Figure 9. A carotenoid-porphyrin-quinone triad exhibiting intramolecular excited-state PCET. ${ }^{66}$ 
Photoinduced electron transfer in molecular dyads and triads often leads to long-lived chargeseparated states in which light energy is transiently stored in the form of chemical energy (an electron-hole pair). ${ }^{64}$ One of the challenges is to obtain charge-separated states which are as long lived as possible, and this provided the motivation for a series of studies presented below.

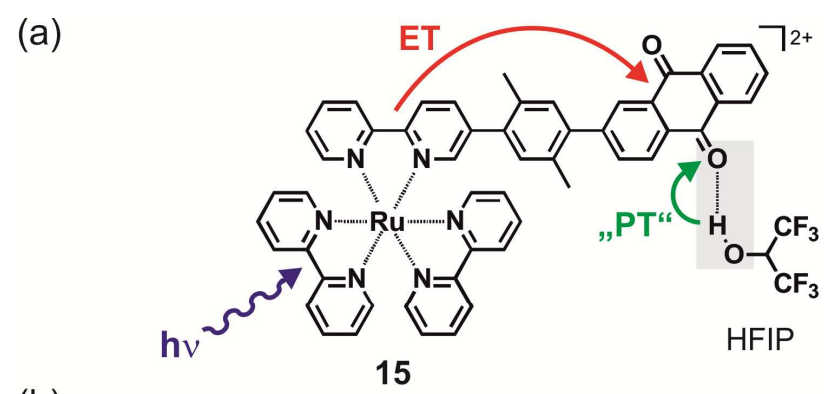

(b)

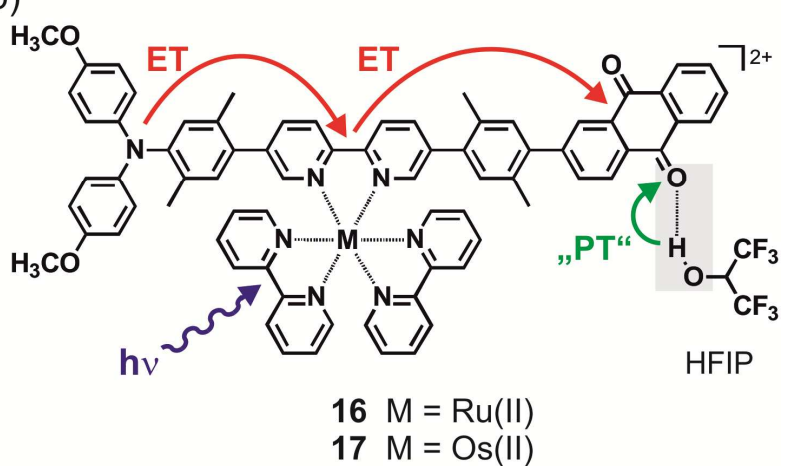

Figure 10. Dyad (a) and triads (b) in which photoproduced anthraquinone radical anions are stabilized by hydrogen-bonds donated by hexafluoroisopropanol (HFIP). ${ }^{69-74}$ H-bonds are strengthened in the course of photoinduced ET, hence „PT” denotes the transfer of some finite proton density rather than the transfer of a full proton.

In dyad 15 photoinduced ET from $\mathrm{Ru}(\mathrm{bpy})_{3}{ }^{2+}$ to anthraquinone (AQ) is endergonic in $\mathrm{CH}_{2} \mathrm{Cl}_{2}$ and consequently does not occur at appreciable rates. ${ }^{69,70}$ However, addition of $3 \mathrm{mM}$ hexafluoroisopropanol (HFIP) makes ET a competitive excited-state deactivation process (Figure 10a). Electrochemical investigations lead to the conclusion that reduction of AQ to AQ- 
increases the average number of HFIP molecules which are hydrogen-bonded to the anthraquinone moiety of dyad $\mathbf{1 5}$ from 1 to 2.5 , and at the same time the (mean) binding constant per HFIP molecule increases from $\sim 1 \mathrm{M}^{-1}$ to $\sim 66 \mathrm{M}^{-1} .{ }^{69}$ In other words, not only more HFIP molecules bind to $\mathrm{AQ}^{-}$than to charge-neutral $\mathrm{AQ}$, but individual HFIP species also bind more tightly. Photoreduction of AQ in dyad $\mathbf{1 5}$ may therefore be regarded as a variant of excited-state PCET: Hydrogen bonds are strengthened in the course of photoinduced ET, implying that some finite proton density (rather than a full proton) is transferred from HFIP toward AQ. Whether or not hydrogen-bond strengthening takes place in concert with AQ (photo)reduction is not clear; at any rate no significant H/D KIEs were detected.
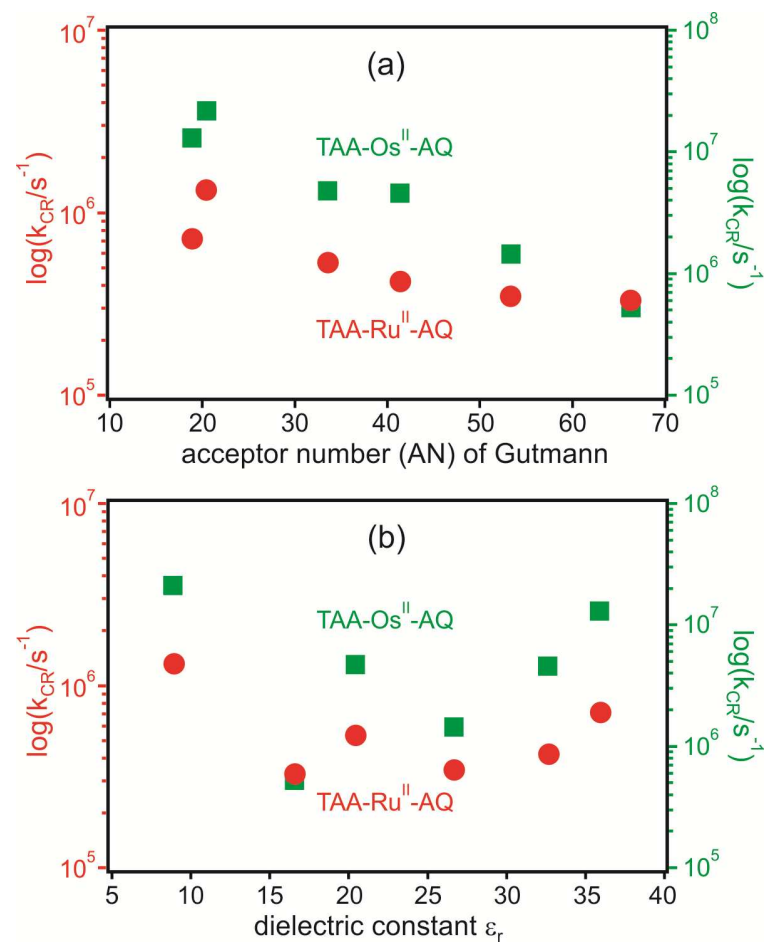

Figure 11. Rate constant for electron-hole recombination $\left(\mathrm{k}_{\mathrm{CR}}\right)$ after photoexcitation of triads 16 (red) and 17 (green) as a function of (a) Gutmann acceptor number of the solvent, (b) dielectric 
constant of the solvent. Adapted with permission from ref. ${ }^{71}$. Copyright 2012 American Chemical Society.

Triads 16 and 17 represent an extension of dyad 15 in that they contain an additional triarylamine (TAA) donor (Figure 10b). ${ }^{72}$ Photoexcitation of their Ru(bpy) ${ }_{3}{ }^{2+}$ and Os(bpy) ${ }_{3}{ }^{2+}$ moieties leads to the rapid formation $(<10 \mathrm{~ns})$ of a charge-separated state containing oxidized TAA and reduced AQ. ${ }^{71,73}$ The decay time $\left(\mathrm{k}_{\mathrm{CR}}\right)$ of this charge-separated state correlates with the Gutmann acceptor number of the solvent in which it is measured (Figure 11a), while the correlation of $\mathrm{k}_{\mathrm{CR}}$ with the solvent dielectric constant is poor (Figure $11 \mathrm{~b}$ ). ${ }^{71}$ These observations suggest that the electron/hole pair is stabilized by H-bond donation from the solvent. The most acidic solvent used in this study was HFIP, and it seems that photoreduction of AQ is coupled to H-bond strengthening rather than to the transfer of a full proton; transient absorption data are consistent with this interpretation. In the case of the osmium triad (17) the change in solvent from $\mathrm{CH}_{2} \mathrm{Cl}_{2}$ to HFIP increased the lifetime of the charge-separated state from $\sim 50$ to 2000 ns. ${ }^{71,74}$ Interestingly, the rates for photoinduced forward processes, specifically AQ reduction, were much less affected by the change in solvent. Furthermore HFIP seems to have little influence on the redox chemistry of TAA. The main conclusion from these recent works and related prior studies is that photoproduced charge-separated states can be stabilized thermodynamically and kinetically by hydrogen-bonding, and this might be of interest for lightto-chemical energy conversion. 


\section{CONCLUDING REMARKS}

Light pulses have been used quite frequently to trigger PCET reactions, but the number of true excited-state PCET systems with photoexcited reactants is small compared to studies of PCET between molecules which are in their electronic ground states. In principle, excited-state PCET offers the possibility for direct conversion of light energy into chemical energy, but appropriate catalytic energy conversion cycles are yet to be successfully implemented. Investigations of excited-state PCET need not be limited to metal complexes with long-lived triplet excited states. Given sufficiently high reaction rates and fast detection techniques, purely organic reagents are amenable to studies of excited-state PCET as well. ${ }^{24}$

Laser-triggering is clearly an interesting tool for investigation of rapid PCETs, and the results obtained from studies of excited-state PCET can be understood in the same theoretical framework as ground-state PCET reactions. However, the sub-discipline of excited-state PCET often suffers from the fact that thermodynamic quantities such as redox potentials and $\mathrm{pK}_{\mathrm{a}}$ values for the relevant photoexcited species can only be estimated.

Solar energy conversion will not necessarily have to rely on excited-state PCET. For example, the PCET reactions of photosystem II involve species in their electronic ground states. Indeed, ground-state PCET might be an equally feasible way to convert solar energy as excited-state PCET. On the other hand, electronically excited species are usually more reactive than molecules in their ground states, and this might pave the way to more unusual PCET reactions than those which have been explored to date. 


\section{BIOGRAPHICAL INFORMATION}

Oliver S. Wenger received a Ph. D. degree from the University of Berne (Switzerland). He was a postdoc at Caltech from 2002 to 2004, and in Strasbourg (France) between 2004 and 2006. Since 2006 Wenger performs independent research, until 2009 as an assistant professor at the University of Geneva, and from 2009 to 2012 as an associate professor (W2) at Georg-AugustUniversität Göttingen (Germany). Since fall 2012 he is professor of inorganic chemistry at the University of Basel (Switzerland).

\section{ACKNOWLEDGMENT}

The author thanks his collaborators and colleagues for their work cited in this Account. Funding from the Swiss NSF through grant number PP002-110611 and by the DFG through grant number WE4815/1-1 and through IRTG-1422 is gratefully acknowledged.

\section{REFERENCES}

(1) Meyer, T. J. Chemical Approaches to Artificial Photosynthesis. Acc. Chem. Res. 1989, 22, 163-170.

(2) Meyer, T. J.; Huynh, M. H. V.; Thorp, H. H. The Possible Role of ProtonCoupled Electron Transfer (PCET) in Water Oxidation by Photosystem II. Angew. Chem. Int. Ed. 2007, 46, 5284-5304. 
(3) Howard, J. B.; Rees, D. C. How Many Metals Does it Take to Fix $\mathrm{N}_{2}$ ? A Mechanistic Overview of Biological Nitrogen Fixation. Proc. Natl. Acad. Sci. U. S. A. 2006, 103, 17088-17093.

(4) Mayer, J. M. Proton-Coupled Electron Transfer: A Reaction Chemist's View. Annu. Rev. Phys. Chem. 2004, 55, 363-390.

(5) Warren, J. J.; Tronic, T. A.; Mayer, J. M. Thermochemistry of Proton-Coupled Electron Transfer Reagents and its Implications. Chem. Rev. 2010, 110, 6961-7001.

(6) Magnuson, A.; Anderlund, M.; Johansson, O.; Lindblad, P.; Lomoth, R.; Polivka, T.; Ott, S.; Stensjö, K.; Styring, S.; Sundström, V.; Hammarström, L. Biomimetic and Microbial Approaches to Solar Fuel Generation. Acc. Chem. Res. 2009, 42, 1899-1909.

(7) Dempsey, J. L.; Winkler, J. R.; Gray, H. B. Proton-Coupled Electron Flow in Protein Redox Machines. Chem. Rev. 2010, 110, 7024-7039.

(8) Costentin, C.; Robert, M.; Savéant, J.-M. Concerted Proton-Electron Transfers: Electrochemical and Related Approaches. Acc. Chem. Res. 2010, 43, 1019-1029.

(9) Hammes-Schiffer, S. Theory of Proton-Coupled Electron Transfer in Energy Conversion Processes. Acc. Chem. Res. 2009, 42, 1881-1889.

(10) Weinberg, D. R.; Gagliardi, C. J.; Hull, J. F.; Murphy, C. F.; Kent, C. A.; Westlake, B. C.; Paul, A.; Ess, D. H.; McCafferty, D. G.; Meyer, T. J. Proton-Coupled Electron Transfer. Chem. Rev. 2012, 112, 4016-4093. 
(11) Rhile, I. J.; Markle, T. F.; Nagao, H.; DiPasquale, A. G.; Lam, O. P.; Lockwood, M. A.; Rotter, K.; Mayer, J. M. Concerted Proton-Electron Transfer in the Oxidation of Hydrogen-Bonded Phenols. J. Am. Chem. Soc. 2006, 128, 6075-6088.

(12) Schrauben, J. N.; Cattaneo, M.; Day, T. C.; Tenderholt, A. L.; Mayer, J. M. Mutiple-Site Concerted Proton-Electron Transfer Reactions of Hydrogen-Bonded Phenols are Nonadiabatic and Well Described by Semiclassical Marcus Theory. J. Am. Chem. Soc. 2012, $134,16635-16645$.

(13) Bonin, J.; Costentin, C.; Louault, C.; Robert, M.; Savéant, J. M. Water (in Water) as an Intrinsically Efficient Proton Acceptor in Concerted Proton Electron Transfers. J. Am. Chem. Soc. 2011, 133, 6668-6674.

(14) Costentin, C.; Robert, M.; Savéant, J. M. Concerted Proton-Electron Transfers in the Oxidation of Phenols. Phys. Chem. Chem. Phys. 2010, 12, 11179-11190.

(15) Irebo, T.; Zhang, M.-T.; Markle, T. F.; Scott, A. M.; Hammarström, L. Spanning Four Mechanistic Regions of Intramolecular Proton-Coupled Electron Transfer in a Ru(bpy) ${ }_{3}{ }^{2+}$ Tyrosine Complex. J. Am. Chem. Soc. 2012, 134, 16247-16254.

(16) Markle, T. F.; Rhile, I. J.; DiPasquale, A. G.; Mayer, J. M. Probing Concerted Proton-Electron Transfer in Phenol-Imidazoles. Proc. Natl. Acad. Sci. U. S. A. 2008, 105, 81858190.

(17) Magnuson, A.; Berglund, H.; Korall, P.; Hammarström, L.; Åkermark, B.; Styring, S.; Sun, L. C. Mimicking Electron Transfer Reactions in Photosystem II: Synthesis and 
Photochemical Characterization of a Ruthenium(II) Tris(bipyridyl) Complex with a Covalently Linked Tyrosine. J. Am. Chem. Soc. 1997, 119, 10720-10725.

(18) Lachaud, T.; Quaranta, A.; Pellegrin, Y.; Dorlet, P.; Charlot, M. F.; Un, S.; Leibl, W.; Aukauloo, A. A Biomimetic Model of the Electron Transfer between P-680 and the TyrZHis 190 Pair of PSII. Angew. Chem. Int. Ed. 2005, 44, 1536-1540.

(19) Biczok, L.; Gupta, N.; Linschitz, H. Coupled Electron-Proton Transfer in Interactions of Triplet C-60 with Hydrogen-Bonded Phenols: Effects of Solvation, Deuteration, and Redox Potentials. J. Am. Chem. Soc. 1997, 119, 12601-12609.

(20) Moore, G. F.; Hambourger, M.; Gervaldo, M.; Poluektov, O. G.; Rajh, T.; Gust, D.; Moore, T. A.; Moore, A. L. A Bioinspired Construct that Mimics the Proton Coupled Electron Transfer between $\mathrm{P}_{680^{+}}$and the $\mathrm{Tyr}_{\mathrm{z}}-\mathrm{His} 190$ Pair of Photosystem II. J. Am. Chem. Soc. 2008, 130, 10466-10467.

(21) Bonin, J.; Costentin, C.; Robert, M.; Savéant, J. M. Pyridine as Proton Acceptor in the Concerted Proton Electron Transfer Oxidation of Phenol. Org. Biomol. Chem. 2011, 9, 4064-4069.

(22) Irebo, T.; Reece, S. Y.; Sjödin, M.; Nocera, D. G.; Hammarström, L. ProtonCoupled Electron Transfer of Tyrosine Oxidation: Buffer Dependence and Parallel Mechanisms. J. Am. Chem. Soc. 2007, 129, 15462-15464.

(23) Gagliardi, C. J.; Westlake, B. C.; Kent, C. A.; Paul, J. J.; Papanikolas, J. M.; Meyer, T. J. Integrating Proton Coupled Electron Transfer (PCET) and Excited States. Coord. Chem. Rev. 2010, 254, 2459-2471. 
(24) Westlake, B. C.; Brennaman, M. K.; Concepcion, J. J.; Paul, J. J.; Bettis, S. E.; Hampton, S. D.; Miller, S. A.; Lebedeva, N. V.; Forbes, M. D. E.; Moran, A. M.; Meyer, T. J.; Papanikolas, J. M. Concerted Electron-Proton Transfer in the Optical Excitation of HydrogenBonded Dyes. Proc. Natl. Acad. Sci. U. S. A. 2011, 108, 8554-8558.

(25) Wenger, O. S. Proton-Coupled Electron Transfer Originating from Excited States of Luminescent Transition-Metal Complexes. Chem.-Eur. J. 2011, 17, 11692-11702.

(26) Balzani, V. Electron Transfer in Chemistry; VCH Wiley: Weinheim, 2001; Vol. 3.

(27) Kirby, J. P.; Roberts, J. A.; Nocera, D. G. Significant Effect of Salt Bridges on Electron Transfer. J. Am. Chem. Soc. 1997, 119, 9230-9236.

(28) Roberts, J. A.; Kirby, J. P.; Nocera, D. G. Photoinduced Electron-Transfer within a Donor-Acceptor Pair Juxtaposed by a Salt Bridge. J. Am. Chem. Soc. 1995, 117, 8051-8052.

(29) Mayer, J. M.; Rhile, I. J. Thermodynamics and Kinetics of Proton-Coupled Electron Transfer: Stepwise vs. Concerted Pathways. Biochim. Biophys. Acta 2004, 1655, 51-58.

(30) Roth, J. P.; Yoder, J. C.; Won, T. J.; Mayer, J. M. Application of the Marcus Cross Relation to Hydrogen Atom Transfer Reactions. Science 2001, 294, 2524-2526.

(31) Freys, J. C.; Bernardinelli, G.; Wenger, O. S. Proton-Coupled Electron Transfer from a Luminescent Excited State. Chem. Commun. 2008, 4267-4269.

(32) Freys, J. C.; Wenger, O. S. Supramolecular and Intramolecular Energy Transfer with Ruthenium-Anthracene Donor-Acceptor Couples: Salt Bridge versus Covalent Bond. Eur. J. Inorg. Chem. 2010, 5509-5516. 
(33) Freys, J. C.; Hanss, D.; Walther, M. E.; Wenger, O. S. Chemistry with Photons, Protons, and Electrons. Chimia 2009, 63, 49-53.

(34) Haga, M.-A. Synthesis and Protonation-Deprotonation Reactions of Ruthenium(II) Complexes Containing 2,2'-Bibenzimidazole and Related Ligands. Inorg. Chim. Acta 1983, 75, 29-35.

(35) Hönes, R.; Kuss-Petermann, M.; Wenger, O. S. Photochemistry between a Ruthenium(II) Pyridylimidazole Complex and Benzoquinone: Simple Electron Transfer versus Proton-Coupled Electron Transfer. Photochem. Photobiol. Sci. 2012, doi: 10.1039/c2pp25270h.

(36) Lancaster, K. M.; Gerken, J. B.; Durrell, A. C.; Palmer, J. H.; Gray, H. B. Electronic Structures, Photophysical Properties, and Electrochemistry of Ruthenium(II)(bpy) 2 Pyridylimidazole Complexes. Coord. Chem. Rev. 2010, 254, 1803-1811.

(37) Wu, A.; Mayer, J. M. Hydrogen Atom Transfer Reactions of a Ruthenium Imidazole Complex: Hydrogen Tunneling and the Applicability of the Marcus Cross Relation. $J$. Am. Chem. Soc. 2008, 130, 14745-14754.

(38) Venturi, M.; Mulazzani, Q. G.; Ciano, M.; Hoffman, M. Z. Radiolytic and Electrochemical Reduction of $\mathrm{Ru}(\mathrm{bpz})_{3}{ }^{2+}$ in Aqueous Solution - Stability, Redox, and Acid-Base Properties of $\mathrm{Ru}(\mathrm{bpz})_{3}{ }^{+}$. Inorg. Chem. 1986, 25, 4493-4498.

(39) Concepcion, J. J.; Brennaman, M. K.; Deyton, J. R.; Lebedeva, N. V.; Forbes, M. D. E.; Papanikolas, J. M.; Meyer, T. J. Excited-State Quenching by Proton-Coupled Electron Transfer. J. Am. Chem. Soc. 2007, 129, 6968-6969. 
(40) Lebedeva, N. V.; Schmidt, R. D.; Concepcion, J. J.; Brennaman, M. K.; Stanton, I. N.; Therien, M. J.; Meyer, T. J.; Forbes, M. D. E. Structural and pH Dependence of Excited State PCET Reactions involving Reductive Quenching of the MLCT Excited State of Ru(bpy $)_{2}(\mathrm{bpz})^{2+}$ by Hydroquinones. J. Phys. Chem. A 2011, 115, 3346-3356.

(41) Bronner, C.; Wenger, O. S. Kinetic Isotope Effects in Reductive Excited-State Quenching of Ru(2,2'-bipyrazine $)_{3}{ }^{2+}$ by Phenols. J. Phys. Chem. Lett. 2012, 3, 70-74.

(42) An e-mail communication with Ian Rhile is much appreciated regarding the interpretation of these data.

(43) Bronner, C.; Wenger, O. S. Proton-Coupled Electron Transfer between 4Cyanophenol and Photoexcited Rhenium(I) Complexes with Different Protonatable Sites. Inorg. Chem. 2012, 51, 8275-8283.

(44) Stewart, D. J.; Brennaman, M. K.; Bettis, S. E.; Wang, L.; Binstead, R. A.; Papanikolas, J. M.; Meyer, T. J. Competing Pathways in the photo-Proton-Coupled Electron Transfer Reduction of fac-Re(bpy) $(\mathrm{CO})_{3}\left(4,4^{\prime}-\mathrm{bpy}\right)^{+*}$ by Hydroquinone. J. Phys. Chem. Lett. 2011, 2, 1844-1848.

(45) Cape, J. L.; Bowman, M. K.; Kramer, D. M. Reaction Intermediates of Quinol Oxidation in a Photoactivatable System that Mimics Electron Transfer in the Cytochrome bc $\mathrm{c}_{1}$ Complex. J. Am. Chem. Soc. 2005, 127, 4208-4215.

(46) Stubbe, J.; Nocera, D. G.; Yee, C. S.; Chang, M. C. Y. Radical Initiation in the Class I Ribonucleotide Reductase: Long-Range Proton-Coupled Electron Transfer? Chem. Rev. 2003, 103, 2167-2201. 
(47) Su, Q. J.; Klinman, J. P. Probing the Mechanism of Proton Coupled Electron Transfer to Dioxygen: The Oxidative Half-Reaction of Bovine Serum Amine Oxidase. Biochemistry 1998, 37, 12513-12525.

(48) Warren, J. J.; Winkler, J. R.; Gray, H. B. Redox Properties of Tyrosine and Related Molecules. FEBS Lett. 2012, 586, 596-602.

(49) Barry, B. A.; Babcock, G. T. Tyrosine Radicals are Involved in the Photosynthetic Oxygen-Evolving System. Proc. Natl. Acad. Sci. U. S. A. 1987, 84, 7099-7103.

(50) Benisvy, L.; Bittl, R.; Bothe, E.; Garner, C. D.; McMaster, J.; Ross, S.; Teutloff, C.; Neese, F. Phenoxyl Radicals Hydrogen-Bonded to Imidazolium: Analogues of Tyrosyl D of Photosystem II: High-Field EPR and DFT Studies. Angew. Chem. Int. Ed. 2005, 44, 5314-5317.

(51) Sun, L. C.; Burkitt, M.; Tamm, M.; Raymond, M. K.; Abrahamsson, M.; LeGourriérec, D.; Frapart, Y.; Magnuson, A.; Kenéz, P. H.; Brandt, P.; Tran, A.; Hammarström, L.; Styring, S.; Åkermark, B. Hydrogen-Bond Promoted Intramolecular Electron Transfer to Photogenerated Ru(III): A Functional Mimic of Tyrosine(Z) and Histidine 190 in Photosystem II. J. Am. Chem. Soc. 1999, 121, 6834-6842.

(52) Reece, S. Y.; Nocera, D. G. Direct Tyrosine Oxidation Using the MLCT Excited States of Rhenium Polypyridyl Complexes. J. Am. Chem. Soc. 2005, 127, 9448-9458.

(53) Zhang, M. T.; Hammarström, L. Proton-Coupled Electron Transfer from Tryptophan: A Concerted Mechanism with Water as Proton Acceptor. J. Am. Chem. Soc. 2011, $133,8806-8809$. 
(54) Pizano, A. A.; Yang, J. L.; Nocera, D. G. Photochemical Tyrosine Oxidation with a Hydrogen-Bonded Proton Acceptor by Bidirectional Proton-Coupled Electron Transfer. Chem. Sci. 2012, 3, 2457-2461.

(55) Fecenko, C. J.; Thorp, H. H.; Meyer, T. J. The Role of Free Energy Change in Coupled Electron-Proton Transfer. J. Am. Chem. Soc. 2007, 129, 15098-15099.

(56) Manner, V. W.; DiPasquale, A. G.; Mayer, J. M. Facile Concerted ProtonElectron Transfers in a Ruthenium Terpyridine-4'-Carboxylate Complex with a Long Distance between the Redox and Basic Sites. J. Am. Chem. Soc. 2008, 130, 7210-7211.

(57) Markle, T. F.; Rhile, I. J.; Mayer, J. M. Kinetic Effects of Increased Proton Transfer Distance on Proton-Coupled Oxidations of Phenol-Amines. J. Am. Chem. Soc. 2011, $133,17341-17352$.

(58) Zhang, M.-T.; Irebo, T.; Johansson, O.; Hammarström, L. Proton-Coupled Electron Transfer from Tyrosine: A Strong Rate Dependence on Intramolecular Proton Transfer Distance. J. Am. Chem. Soc. 2011, 133, 13224-13227.

(59) Kuss-Petermann, M.; Wolf, H.; Stalke, D.; Wenger, O. S. Influence of DonorAcceptor Distance Variation on Photoinduced Electron and Proton Transfer in Rhenium(I)Phenol Dyads. J. Am. Chem. Soc. 2012, 134, 12844-12854.

(60) Tolbert, L. M.; Solntsev, K. M. Excited-State Proton Transfer: From Constrained Systems to "Super" Photoacids to Superfast Proton Transfer. Acc. Chem. Res. 2002, 35, 19-27. 
(61) Irebo, T.; Johansson, O.; Hammarström, L. The Rate Ladder of Proton-Coupled Tyrosine Oxidation in Water: A Systematic Dependence on Hydrogen Bonds and Protonation State. J. Am. Chem. Soc. 2008, 130, 9194-9195.

(62) Gupta, N.; Linschitz, H. Hydrogen-Bonding and Protonation Effects in Electrochemistry of Quinones in Aprotic Solvents. J. Am. Chem. Soc. 1997, 119, 6384-6391.

(63) Fukuzumi, S.; Okamoto, K.; Yoshida, Y.; Imahori, H.; Araki, Y.; Ito, O. Effects of Hydrogen Bonding on Metal Ion-Promoted Intramolecular Electron Transfer and Photoinduced Electron Transfer in a Ferrocene-Quinone Dyad with a Rigid Amide Spacer. $J$. Am. Chem. Soc. 2003, 125, 1007-1013.

(64) Wasielewski, M. R. Photoinduced Electron-Transfer in Supramolecular Systems for Artificial Photosynthesis. Chem. Rev. 1992, 92, 435-461.

(65) Yago, T.; Gohdo, M.; Wakasa, M. Hydrogen Bonding Effects on the Reorganization Energy for Photoinduced Charge Separation Reaction between Porphyrin and Quinone Studied by Nanosecond Laser Flash Photolysis. J. Phys. Chem. B 2010, 114, 24762483.

(66) Hung, S. C.; Macpherson, A. N.; Lin, S.; Liddell, P. A.; Seely, G. R.; Moore, A. L.; Moore, T. A.; Gust, D. Coordinated Photoinduced Electron and Proton-Transfer in a Molecular Triad. J. Am. Chem. Soc. 1995, 117, 1657-1658.

(67) Steinberg-Yfrach, G.; Liddell, P. A.; Hung, S. C.; Moore, A. L.; Gust, D.; Moore, T. A. Conversion of Light Energy to Proton Potential in Liposomes by Artificial Photosynthetic Reaction Centres. Nature 1997, 385, 239-241. 
(68) Biczok, L.; Linschitz, H. Oxidation of Triplet C-60 by Hydrogen-Bonded Chloranil: Efficient Formation, Spectrum and Charge-Shift Reactions of C- $60^{+}$Cation Radical. $J$. Phys. Chem. A 2001, 105, 11051-11056.

(69) Hankache, J.; Hanss, D.; Wenger, O. S. Hydrogen-Bond Strengthening upon Photoinduced Electron Transfer in Ruthenium-Anthraquinone Dyads Interacting with Hexafluoroisopropanol or Water. J. Phys. Chem. A 2012, 116, 3347-3358.

(70) Hankache, J.; Wenger, O. S. Photoinduced Electron Transfer in Covalent Ruthenium-Anthraquinone Dyads: Relative Importance of Driving-Force, Solvent Polarity, and Donor-Bridge Energy Gap. Phys. Chem. Chem. Phys. 2012, 14, 2685-2692.

(71) Hankache, J.; Wenger, O. S. Hydrogen-Bonding Effects on the Formation and Lifetimes of Charge-Separated States in Molecular Triads. J. Phys. Chem. A 2012, 116, 81598168.

(72) Hankache, J.; Wenger, O. S. Microsecond Charge Recombination in a Linear Triarylamine-Ru(bpy) ${ }_{3}{ }^{2+}$-Anthraquinone Triad. Chem. Commun. 2011, 47, 10145-10147.

(73) Hankache, J.; Niemi, M.; Lemmetyinen, H.; Wenger, O. S. Photoinduced Electron Transfer in Linear Triarylamine-Photosensitizer-Anthraquinone Triads with Ruthenium(II), Osmium(II), and Iridium(III). Inorg. Chem. 2012, 51, 6333-6344.

(74) Hankache, J.; Wenger, O. S. Large Increase of the Lifetime of a Charge-Separated State in a Molecular Triad Induced by Hydrogen-Bonding Solvent. Chem. Eur. J. 2012, 18, 6443-6447. 